\title{
Saturation of the laser-induced narrowband coherent synchrotron radiation process: Experimental observation at a storage ring
}

\author{
M. Hosaka, N. Yamamoto, and Y. Takashima \\ Graduate School of Engineering, Nagoya University, Nagoya, 464-8603, Japan \\ C. Szwaj, ${ }^{1,2}$ M. Le Parquier, ${ }^{2}$ C. Evain, ${ }^{1,2}$ and S. Bielawski ${ }^{1,2}$ \\ ${ }^{1}$ Laboratoire de Physique des Lasers, Atomes et Molécules, UMR CNRS 8523, Université des Sciences et Technologies de Lille, \\ F-59655 Villeneuve d'Ascq Cedex, France \\ ${ }^{2}$ Centre d'Études et de Recherches Lasers et Applications, FR CNRS 2416, \\ Université des Sciences et Technologies de Lille, F-59655 Villeneuve d'Ascq Cedex, France \\ M. Adachi, H. Zen, T. Tanikawa, S. Kimura, and M. Katoh \\ UVSOR Facility, Institute for Molecular Science, National Institutes of Natural Sciences, Okazaki 444-8585, Japan \\ M. Shimada \\ High Energy Accelerator Research Organization, KEK, Tsukuba, 305-0801, Japan \\ T. Takahashi \\ Research Reactor Institute, Kyoto University, 590-0494, Japan \\ (Received 5 January 2012; revised manuscript received 20 November 2012; published 1 February 2013)
}

\begin{abstract}
We study the efficiency limitation affecting laser-induced coherent synchrotron radiation (CSR) at high laser power. Experiments are made on the UVSOR-II storage ring in conditions of narrowband terahertz CSR emission. While, at moderate power, CSR power increases quadratically with laser power, a noticeable decrease in efficiency and eventually a decrease in CSR power is observed experimentally at high power. Details of the underlying process are analyzed numerically. As the saturation effect depends almost instantaneously on the laser intensity, the saturation occurs locally in longitudinal space. This has important consequences on the modulation pattern induced on the electron bunch.
\end{abstract}

DOI: 10.1103/PhysRevSTAB.16.020701

PACS numbers: 42.65.Ky, 41.60.Ap, 41.75.Ht

In a bending magnet, a relativistic electron bunch can radiate coherent synchrotron radiation (CSR) in the terahertz domain when its longitudinal charge density distribution presents Fourier components at terahertz frequencies. This happens in short (typically picosecond) electron bunches [1,2], but also in long electron bunches in which a longitudinal modulation has been performed. Such modulated bunch experiments have been realized in the conditions of slicing [3-8], in which an external laser creates a picosecond/subpicosecond "dip" in the longitudinal charge distribution, leading to broadband short terahertz emission. It is also possible to obtain narrowband emission with a similar setup, if a quasisinusoidal modulation is performed on the electron bunch [6,9-15].

Detailed asymptotic studies [11] of the efficiency were performed in the case where the electron energy is modulated in an undulator. Emitted power increases with current and laser power-in the limit of low power-with well-

Published by the American Physical Society under the terms of the Creative Commons Attribution 3.0 License. Further distribution of this work must maintain attribution to the author(s) and the published article's title, journal citation, and DOI. defined polynomial scaling laws. In addition to the wellknown quadratic scaling of emitted power with current [2], the scaling is also quadratic with laser power (i.e., is proportional to the fourth power of density modulation amplitude). However, when the laser power is arbitrarily increased, a departure from this quadratic increase with laser power is also expected, eventually leading to a decrease of emitted power with laser power. This is illustrated in Fig. 1.

In this paper, we report on a direct observation of this saturation effect in the case of narrowband CSR induced by laser, and we numerically analyze the process. The global organization of the experiment at UVSOR-II (Fig. 2) is similar to the one of Ref. [11] (and to typical slicing experiments in general [3-8]). In order to observe the saturation phenomenon in the long-pulse regime, we used higher energy pulses (by means of a new amplifier), and realized a pulse shaper able to provide modulated pulses with adjustable duration and modulation period (see Fig. 3). The laser system is an amplified titanium-sapphire laser (composed of a Mira, Legend, and Hidra from Coherent Inc.), able to deliver $50 \mathrm{~mJ}$ pulses of $130 \mathrm{fs}$ duration. In these experiments, we used the uncompressed output of the amplifier, which provides strongly chirped 


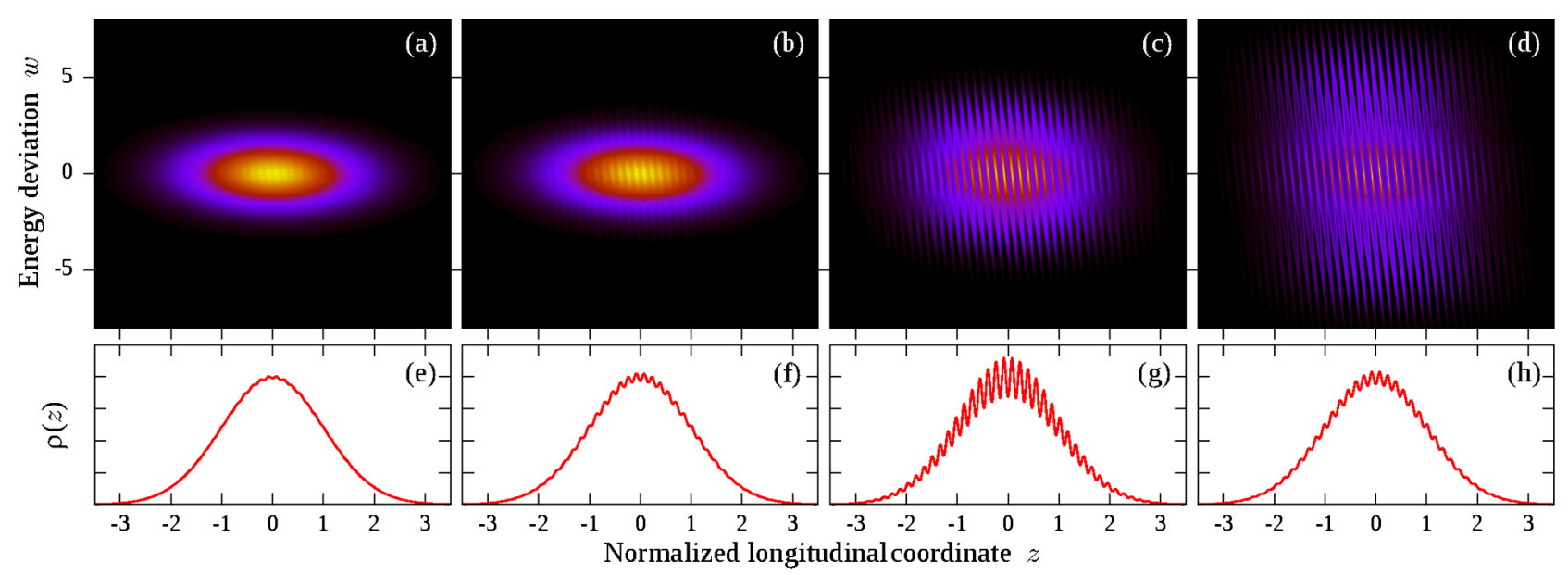

FIG. 1. Illustration of the saturation process in a simplified case where the laser pulse is infinitely long, and the magnetic transport involves only longitudinal dynamics (more precisely $r_{51}=r_{51}=0$ and $r_{56} \neq 0$ ). The upper [(a)-(d)] and lower rows [(e)-(h)] represent the longitudinal phase space, and the longitudinal density profile $\rho(z)$ respectively, at the emission point for increasing laser power. The tilt of the modulation pattern has a fixed angle determined by the magnetic transport parameter $r_{56}$. The increase in laser power leads to a monotonic increase of energy modulation. However, the modulation amplitude in the vertical projection (or equivalently the bunching factor) increases up to an absolute maximum [from (a) to approximately (c)]. Then the amplitude of the density modulation begins to decrease and remains below this absolute maximum. The model used in this simulation is described in Ref. [11], and the parameters used for the illustration are $r_{56}=0.4, k_{m}=40, w_{0}=0.03$ for (a), $w_{0}=0.06$ for (b), $w_{0}=0.3$ for (c), and $w_{0}=0.6$ for (d). The longitudinal coordinate is expressed in units of the initial bunch length. Calculations made in the paper will be performed including $3 \mathrm{D}$ beam transport and a finite duration laser pulse.

pulses (duration 300 ps FWHM, energy $70 \mathrm{~mJ}$ ) at $10 \mathrm{~Hz}$. We constructed a custom external grating compressor delivering pulses, the duration of which is adjustable in a wide range (from $191 \mathrm{ps}$ to full compression), thus providing another way to adjust the peak power. For this purpose, we placed one of the gratings on a long range translation stage $(60 \mathrm{~cm})$ and also carefully designed the compressor so that the introduced delay was almost independent of the distance between gratings. Quasisinusoidal modulation of the pulses is achieved using the technique of chirped pulse beating [16], involving a Michelson interferometer (see [11] for details). The longitudinal modulation period $T_{m}$ of the laser pulses scales with the following law [16]:

$$
\frac{1}{T_{m}} \approx C \frac{\Delta_{\mathrm{Mich}}}{\left(G_{1} G_{2}-G_{1} G_{2}^{0}\right)},
$$

where $G_{1} G_{2}$ is the distance between gratings, $\Delta_{\text {Mich }}$ is the Michelson path difference, and $C$ is a constant. $G_{1} G_{2}^{0}$ is the value of $G_{1} G_{2}$ associated with perfect compression. This approximation is valid while the pulse duration is much larger than the compressed value ( $\approx 130 \mathrm{fs}$ here) and does not take into account third order dispersion [16]. $G_{1} G_{2}^{0}$ and $C$ are evaluated from the fit of Eq. (1) to the emitted CSR frequencies, for various Michelson delays and grating distances. Once the parameters are known, any modulation frequency and pulse duration can be chosen by driving adequately the translation stages of the Michelson interferometer and compressor.
The storage ring is operated in a single bunch at $600 \mathrm{MeV}$ energy, with $100 \mathrm{ps}$ rms bunch duration. The optical klystron is tuned so that it is resonant with the laser wavelength $(800 \mathrm{~nm})$. The laser is focused in the first undulator using a lens with $5 \mathrm{~m}$ focal length, and power is adjusted using a half-wave plate (HWP) placed in front of a polarizing cube. To ensure that transverse direction of the laser beam is not affected during power adjustment, we use a low-angular-error HWP $(<0.5 \mathrm{arc} \mathrm{sec})$. Absence of noticeable transverse motion was confirmed using the camera system used for transverse adjustment (imaging of the SR and laser beam shapes inside undulator). CSR is monitored using an InSb hot-electron bolometer (QMC $\mathrm{QFI} / 2)$ with $1 \mu$ s response time.

Saturation experiments are performed with a laser pulse modulation period of 2.38 ps (i.e., a terahertz emission spectroscopic wave number of $14 \mathrm{~cm}^{-1}$ ), which corresponds approximately to the maximum efficiency of the system.

In the present conditions, departures from quadratic scaling are typically observed, as is shown in Fig. 4. Near the maximum laser pulse duration available from the pulse shaper (191 ps FWHM), only a slight (though noticeable) departure from quadratic scaling is observed [Fig. 4(b)]. However, when the laser pulses are further compressed [down to 28.5 ps FWHM in Fig. 4(c)], a strong saturation effect is observed, leading to the appearance of a maximum. For reference, let us recall that a quasiperfect quadratic law is observed when $300 \mathrm{ps} 2 \mathrm{~mJ}$ pulses [11] are used [Fig. 4(a)]. 


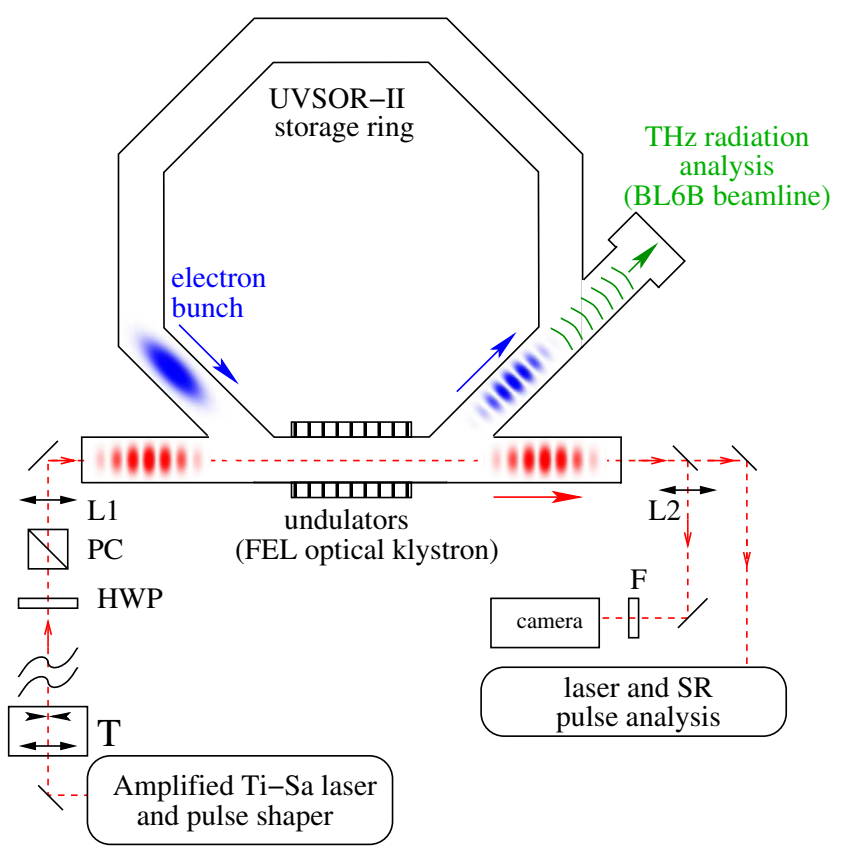

FIG. 2. Global organization of the experiment at the UVSORII storage ring. We produce long laser pulses with an adjustable duration and a sinusoidal intensity modulation. The period is adjustable in the cm-submillimeter range, and the duration is typically chosen from few ps to 190 ps (FWHM). The laser pulses interact with the electron bunch inside an undulator (FEL beam line) and produce an energy modulation. The transport through magnetic elements (in particular dipoles) converts this energy modulation into a charge density modulation, and narrowband CSR terahertz emission is produced at bending magnet beam lines. CSR emission is monitored at the BL6B beam line. T: $0.5 \times$ telescope; HWP: half-wave plate; PC: polarizing cube; L1: $5 \mathrm{~m}$ focusing lens; L2: $2.5 \mathrm{~m}$ imaging lens; F: partially rejecting filter at $800 \mathrm{~nm}$, adjusted to obtain simultaneously synchrotron radiation and laser images with similar intensities on the camera. The imaging system (camera and lens L2) provides an image of laser and SR transverse profiles in the first undulator (typically the center). Details of transport mirrors are not represented.

In order to analyze the process in detail, we use the theoretical approach introduced in the framework of laserinduced CSR [6,11]. A simplification consists of assuming a Gaussian electron bunch distribution and a plane wave for the laser. In this case numerical calculations of the form factor and bunch shape reduce to a simple quadrature. Furthermore, it is possible to derive an asymptotic approximation of the density distribution $\rho(z)$ and its Fourier transform $\tilde{\rho}(k)$, valid when the modulation time scales are much longer than the laser optical carrier time scale (slow amplitude and phase approximation). We have

$$
\rho(z)=\frac{1}{2 \pi} \int_{-\infty}^{+\infty} e^{i k z} \tilde{\rho}(k) d z
$$

$\rho(z)$ and $\tilde{\rho}(k)$ may be approximated by [11]

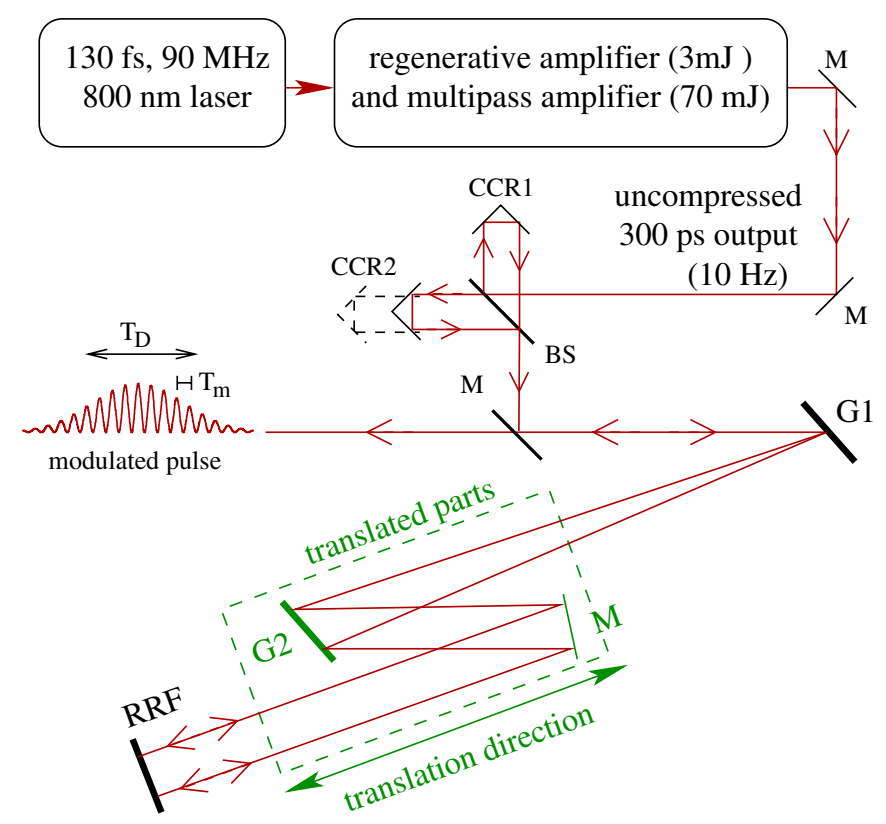

FIG. 3. Laser and pulse-shaper setup. The amplified laser system (from Coherent Inc.) is composed of a Mira oscillator, a Legend regenerative amplifier, and a Hidra multipass amplifier, whose uncompressed (chirped) output is used. The pulse shaper is composed of a Michelson interferometer and an adjustable compressor. The CCR2 Michelson retroreflector is mounted on a motorized translation stage, allowing to adjust the output pulse period $T_{m}$. The pulse duration $T_{D}$ can be changed by adjusting the distance between gratings. The parts displayed in green (mirror and G2 grating) are fixed on the carrier of a single translation stage with wide range $(60 \mathrm{~cm})$. With this configuration, the output pulse delay is not significantly affected by the large displacements of the translation stage. CCR1 and CCR2 are protected gold-coated corner cube retroreflectors with low angular error ( $0.5 \mathrm{arcsec})$. RRF: roof retroreflector; M: mirrors (HR at $800 \mathrm{~nm}$ ); BS: 50\% beam splitter; G1 and G2: 1800 1/mm compression gratings from Spectrogon. Transport mirrors are not represented in detail. The compressor produces pulses whose duration is adjustable from $\approx 130$ fs to 190 ps FWHM.

$$
\begin{aligned}
\tilde{\rho}(k) \approx \tilde{\rho}_{0}(k)= & e^{-\left[\left(r_{51}^{2}+r_{52}^{2}+r_{56}^{2}\right) / 2\right] k^{2}} \\
& \times \int_{-\infty}^{+\infty} e^{-i k z} \frac{e^{-z^{2} / 2}}{\sqrt{2 \pi}} J_{0}\left[k r_{56} a(z)\right] d z,
\end{aligned}
$$

and

$$
\rho(z) \approx \rho_{0}(z)=\frac{1}{2 \pi} \int_{-\infty}^{+\infty} e^{i k z} \tilde{\rho}_{0}(k) d z .
$$

In this expression, all quantities are dimensionless (see Table I, and also Ref. [11] for details). In particular, note that the longitudinal coordinate $z$ is normalized by the electron bunch rms length $\sigma_{z} \cdot a(z)$ characterizes the slowly varying complex amplitude of the laser field. Technically, it corresponds to the local energy modulation induced by the laser, and is expressed in units of the energy spread. Here, we will use essentially 

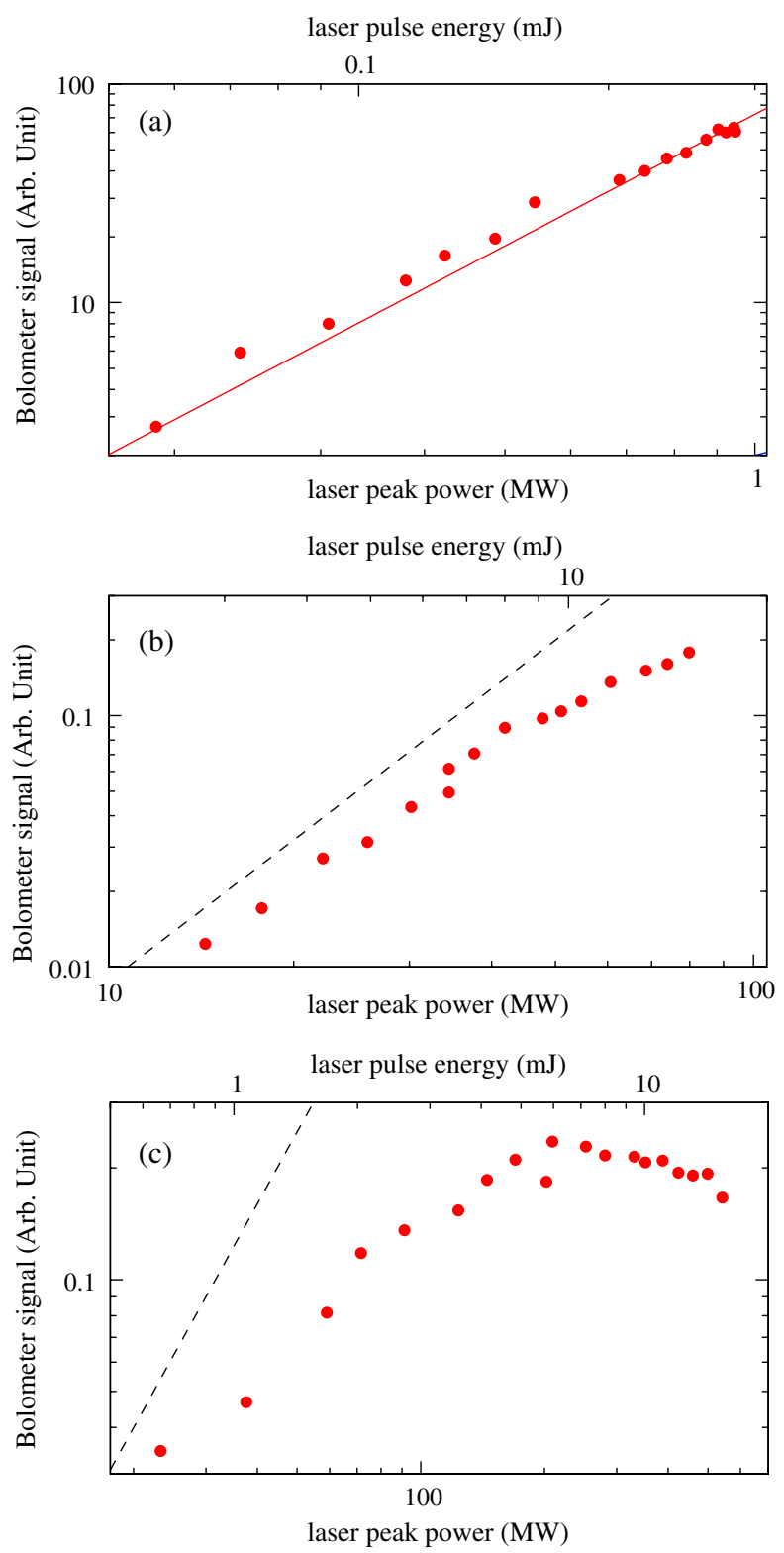

FIG. 4. Typical scalings of terahertz signal versus laser power for three power ranges: (a) reference quasiquadratic behavior that was already observed at low power, with a $2 \mathrm{~mJ}$ amplifier and 300 ps long-pulses (the dots and the full line represent respectively the experimental data and a quadratic fit); (b) and (c) behaviors obtained with the current $70 \mathrm{~mJ}$ amplifier and shorter pulses: 191 ps FWHM (b) and 28.5 ps (c). The dashed lines represent a reference quadratic slope. Modulation period is identical ( $\approx 2.4 \mathrm{ps}$, i.e., $14 \mathrm{~cm}^{-1}$ ) for each recording. Note that electron bunch duration is $\approx 240$ ps FWHM (length $3.1 \mathrm{~cm}$ rms).

$$
a(z)=w_{0} e^{-z^{2} / 4 \sigma_{L}^{2}} \times \cos \left(\frac{k_{m}}{2} z+\phi\right)
$$

with $w_{0}$ the maximum energy modulation amplitude induced by the laser. $k_{m}$ is the modulation wave number of the envelope of the laser pulse, typically in the millimeter/ submillimeter range (note that this is different from the optical carrier of the laser, which is in the micrometer range). $\sigma_{L}$ is the laser pulse rms length. $\phi$ characterizes the phase of the envelope modulation. Note also that $\rho(z)$ and $\rho_{0}(z)$ are normalized so that

$$
\int_{-\infty}^{+\infty} \rho_{0}(z) d z=\int_{-\infty}^{+\infty} \rho(z) d z=1
$$

and

$$
\tilde{\rho}_{0}(k=0)=1
$$

A direct information given by Eq. (4) is the so-called form factor:

$$
f(\bar{\nu})=\left|\tilde{\rho}_{0}(k)\right|^{2}=\left|\tilde{\rho}_{0}\left(2 \pi \bar{\nu} \sigma_{z}\right)\right|^{2},
$$

which gives information on the emitted spectrum [17].

We have calculated $\tilde{\rho}_{0}(k)$ versus the energy modulation $w_{0}$, in the conditions of our experiment, for different laser pulse widths. The integral in Eq. (4) was computed using the Gauss-Kronrod method (DQAGE routine from the QUADPACK library [18]). Then, we have used a representation similar to the experimental one (Fig. 4), where terahertz pulse energy, or more precisely the bolometer signal, was plotted as a function of the laser pulse energy. For this purpose, we have represented the integrated form factor:

$$
F_{\mathrm{int}}=\int_{a}^{b}\left|\tilde{\rho}_{0}(k)\right|^{2} d k,
$$

which is proportional to the terahertz pulse energy, versus $\sigma_{L} w_{0}^{2}$ (approximately proportional to laser pulse energy).

This representation shows that saturation plays an important role in the optimization of the output pulse energy. In Fig. 5, we can see that at low pulse energy, efficiency increases when the pulse duration is decreased. At higher pulse energies, the saturation effect leads to an intersection between the curves (and higher CSR pulse energy may be obtained for longer laser pulses). As a technical consequence, the search for maximum pulse energy may in general require the use of a pulse shaper with adjustable pulse duration.

Furthermore, the data obtained when the saturation effect is strong [Fig. 4(c)] present sufficient features to allow a fit of the theoretical expression of $F_{\text {int }}$ versus $\sigma_{L} w_{0}^{2}$ on the experimental data with two parameters. In our case, one parameter, $C_{1}$, was the ratio between the bolometer signal $V_{L}$ and $F_{\text {int }}$, and the second parameter, $C_{2}$, was the ratio between the square root of the laser pulse energy $Q_{L}$ and $w_{0}^{2}$ :

$$
V_{B}=C_{1} \int_{a}^{b}\left|\tilde{\rho}_{0}(k)\right|^{2} d k,
$$

$$
a(z)=C_{2} \sqrt{Q_{L}} e^{-z^{2} / 4 \sigma_{L}^{2}} \times \cos \left(\frac{k_{m}}{2} z+\phi\right),
$$


TABLE I. Links between physical and dimensionless variables used in the paper. Bunch sizes are rms values just before laser-electron bunch interaction.

\begin{tabular}{lccc}
\hline \hline Quantity name & Physical quantity (unit) & Dimensionless quantity & Relation \\
\hline Bunch length & $\sigma_{z}(\mathrm{~m})$ & 1 & \\
Relative energy spread & $\sigma_{\delta}$ & 1 & \\
Bunch size along $x$ & $\sigma_{x}(\mathrm{~m})$ & 1 & \\
Bunch size along $x^{\prime}$ & $\sigma_{x^{\prime}}$ & 1 & $z=Z / \sigma_{z}$ \\
Longitudinal coordinate & $Z(\mathrm{~m})$ & $z$ & $k=K \sigma_{z}$ \\
Wave number & $K\left(\mathrm{~m}^{-1}\right)$ & $k$ & $k_{m}=K_{m} \sigma_{z}$ \\
Modulation & $K_{m}\left(\mathrm{~m}^{-1}\right)$ & $k_{m}$ & $k=2 \pi \bar{\nu} \sigma_{z}$ \\
Wave number & $=2 \pi c / T_{m}$ & & $k_{m}=2 \pi \bar{\nu}_{m} \sigma_{z}$ \\
Spectroscopic & $\bar{\nu}\left(\mathrm{m}^{-1}\right)$ & & $r_{51}=R_{51} \frac{\sigma_{x}}{\sigma_{z}}$ \\
Wave numbers & $\bar{\nu}_{m}\left(\mathrm{~m}^{-1}\right)$ & $r_{51}$ & $r_{52}=R_{52} \frac{\sigma_{x^{\prime}}}{\sigma_{z}}$ \\
$R_{51}$ & $R_{51}$ & $r_{52}$ & $r_{56}=R_{56} \frac{\sigma_{\delta}}{\sigma_{z}}$ \\
$R_{52}$ & $R_{52}(\mathrm{~m})$ & $r_{56}$ & $a(z)=w(Z) / \sigma_{\delta}$ \\
$R_{56}$ & $R_{56}(\mathrm{~m})$ & $a(z)$ & \\
Energy modulation & $w(Z)$ & & \\
\hline \hline
\end{tabular}

the form factor being calculated as previously using Eqs. (2)-(4). The resulting fit, performed with a classical Levenberg Marquardt algorithm, is represented in Fig. 6. The shape of the calculated curve is in satisfying agreement with the experimental data.

An additional consistency test can also be obtained from the fitted parameter $C_{2}$, giving the dependence between energy modulation and laser power. This dependence can be checked independently using the Zholents and Holldack analytical expressions [19]. For this purpose (see

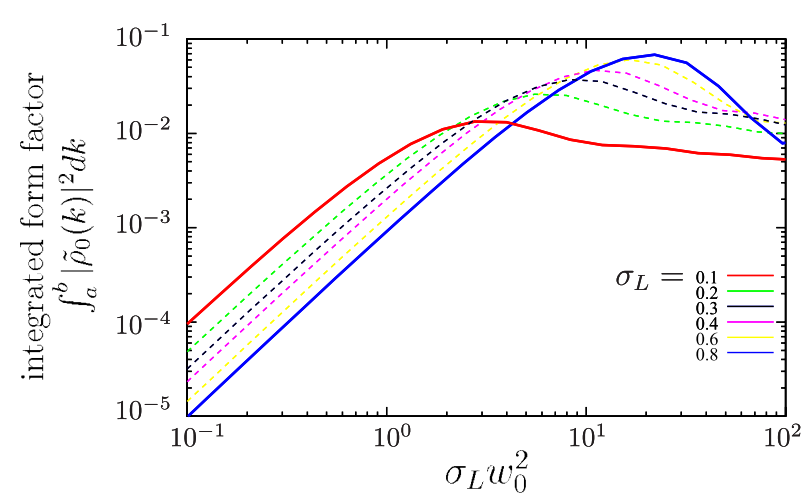

FIG. 5. Numerical calculation of the efficiency, represented in conditions similar to the experimental ones (Fig. 4). Note that dimensionless units are used. The quantity $\sigma_{L} w_{0}^{2}$ is proportional to laser pulse energy. The integrated form factor is proportional to the terahertz pulse energy (and the bolometer signal). The laser pulse duration $\sigma_{L}$ is expressed in units of the electron bunch duration. The two thick full lines $\left(\sigma_{L}=0.1\right.$ and $\sigma_{L}=$ 0.8) approximately correspond to the pulse durations of Fig. 4 (28.5 ps, i.e., $\sigma_{L}=0.117$ and 191 ps, i.e., $\sigma_{L}=0.78$ ). The modulation wave number is $k_{m}=272$, which corresponds to $14 \mathrm{~cm}^{-1}$. We took $\phi=0$, and other parameters are listed in Tables I and II.
Table III), we used the machine and undulator parameters of Ref. [8], a laser pulse duration of 28.5 ps (calculated), and a Rayleigh length of $0.37 \mathrm{~m}$ (calculated from the amplifier characteristics and the transport optics). When the pulse energy is $6 \mathrm{~mJ}$, i.e., at the maximum of Figs. 4(c) and 6 , we find a relative energy modulation of $w_{0}=7.4$ (i.e., 7.4 times the beam energy spread). This value is consistent with the value obtained from the maximum location $\left(w_{0}=5.5\right)$ in Fig. 6. This may be considered as satisfying with respect to previous theory-experiment comparisons [4,5,20], and given the approximations made in the modeling (in particular, the plane wave approximation for the laser).

The second information provided by this fit (the values of $C_{1}$, that are linked to obtain the integrated form factor) should be interpreted with more care. In our experiments the laser beam waist was smaller than the electron bunch,

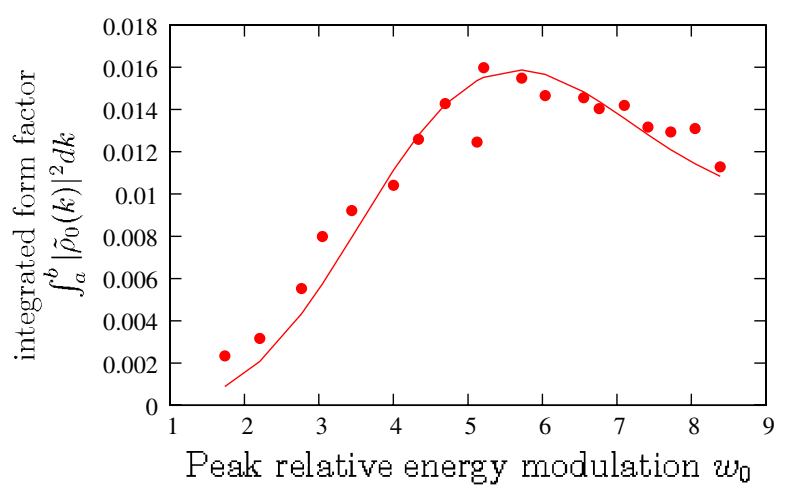

FIG. 6. Least square fit of Eq. (9) on experimental data. $w_{0}$ is the energy modulation in units of the initial energy spread. The vertical values are expected to be overestimated, as the laser beam has a smaller diameter than the electron bunch. Equal weights are attributed to every experimental points. 
TABLE II. Beam and dipole parameters used in the numerical calculations.

\begin{tabular}{lc}
\hline \hline Parameter & Associated dimensionless parameter \\
\hline$\sigma_{x}=4.17 \times 10^{-4} \mathrm{~m}$ & \\
$\sigma_{x^{\prime}}=4.2 \times 10^{-5}$ & \\
$\sigma_{z}=3.1 \times 10^{-2} \mathrm{~m}$ & \\
$\sigma_{\delta}=3.4 \times 10^{-4}$ & \\
$R_{51}=0.0396$ & $r_{51}=0.00053$ \\
$R_{52}=-1.53 \mathrm{~m}$ & $r_{52}=-0.0021$ \\
$R_{56}=-0.268 \mathrm{~m}$ & $r_{56}=-0.0029$ \\
\hline \hline
\end{tabular}

TABLE III. Parameters used in the independent calculation of the energy modulation, using the method described in Ref. [19]. The $K$ value of the undulator (and therefore its resonance wavelength) is tuned for maximum efficiency (see Ref. [19] for details).

\begin{tabular}{lc}
\hline \hline Parameter & Value \\
\hline Rayleigh length & $0.37 \mathrm{~m}$ \\
Laser wavelength & $800 \mathrm{~nm}$ \\
Laser pulse duration & $28.5 \mathrm{ps}$ \\
Laser pulse energy & $6 \mathrm{~mJ}$ \\
Undulator length & $0.99 \mathrm{~m}$ \\
Undulator resonance wavelength & $775 \mathrm{~nm}$ \\
Undulator period & $0.11 \mathrm{~m}$ \\
Beam energy & $600 \mathrm{MeV}$ \\
\hline \hline
\end{tabular}

and only the central part of the electron bunch experiences the modulation. This means that the values of the integrated form factor in Fig. 6 are overestimated, and should not be used directly. Measure of the integrated form factor in our situation will thus require an extension of this theoretical approach (beyond the scope of our paper) to take into account the laser transverse shape. However, we expect the present theory to provide reasonable precision in cases where the laser beam is much wider than the electron bunch itself.

Another feature directly observable in the numerical simulation [simply by making the inverse Fourier transform of $\left.\tilde{\rho}_{0}(k)\right]$ concerns the modulation pattern imprinted on the electron bunch, and in particular its modifications during the saturation process. At low power, the domain of quadratic dependence of CSR power versus laser power is linked to the validity domain of Taylor expansion for the Bessel function $J_{0}\left[k r_{56} a(z)\right]$ in Eq. (4), more precisely

$$
|a(z)| \ll \frac{1}{\left|k_{m} r_{56}\right|} .
$$

For $|a(z)|$ of the order of, or larger than, $1 /\left|k r_{56}\right|$, the damped oscillatory features of the $J_{0}$ function then explain the saturation effect. However, because of the longitudinal dependence of $k r_{56} a(z)$ (i.e., laser power is larger at the

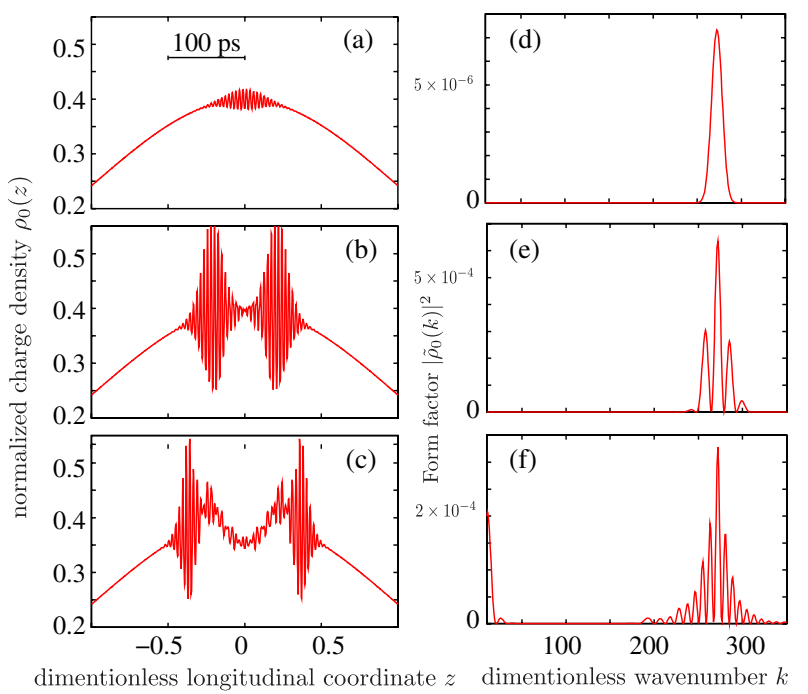

FIG. 7. Local saturation process visible on the normalized charge density profile $\rho_{0}(z)$ of the electron bunch. Numerical calculations were made using $w_{0}=1$ (a), $w_{0}=10$ (b), and $w_{0}=50$ (c). Panels (d), (e), and (f) show the form factors (i.e., power spectra) associated to (a), (b), and (c), respectively. Dimensionless laser pulse duration is $\sigma_{L}=0.117$ (i.e., $28.5 \mathrm{ps}$ ). Other parameters are the same as for Fig. 5.

pulse center than on its edges), the saturation involves a local saturation effect. In Fig. 7, we have represented the longitudinal density distribution of the electron bunch $\rho_{0}(z)$, for different values of the maximum energy modulation $w_{0}$. It clearly appears that a strong decrease of the $\mathrm{THz}$ oscillation occurs near the center, i.e., where laser power is such that $|a(z)| \gg 1 /\left|k_{m} r_{56}\right|$. However, in this situation, strong $\mathrm{THz}$ oscillation of the bunch shape is anyway observed at locations where the laser power is moderate.

A side effect of the local saturation effect concerns the CSR emission spectrum. As observed in Ref. [11], a strong modulation of the CSR peak is observed at high power. As may be seen from Fig. 7, the spectrum modulation may be viewed as a consequence of the special feature of the bunch profile [Figs. 7(a)-7(c)].

In conclusion, it appears that both quadratic dependence of the CSR power and strong saturation may be observed experimentally, with millijoule lasers. A first consequence of this effect concerns efficiency, as saturation affects the dependence of terahertz pulse energy versus laser pulse parameters. From the theoretical point of view, an intuitive local saturation effect is found to affect several aspects of the process. This concerns efficiency curves, in a similar way to the case of coherent harmonic generation [21], and shares common points with the more complex pulse splitting phenomenon in single-pass free-electron lasers (FELs) [22]. Future extensions of this work concern studies of the consequences of this saturation effect in various situations. 


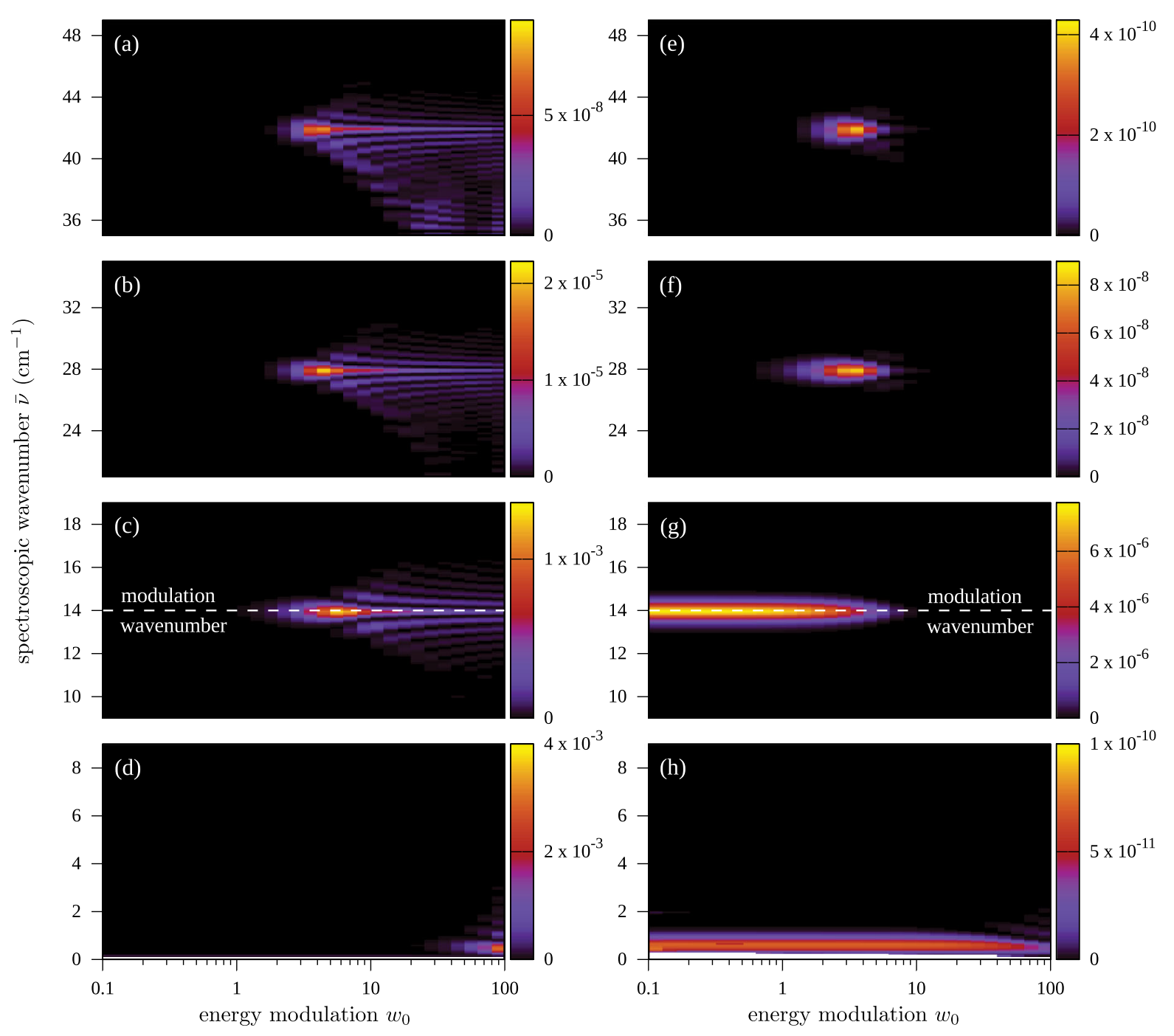

FIG. 8. Form factor versus wave number and energy modulation, for a modulation wave number $k_{m}=272$ (i.e., $\approx 14 \mathrm{~cm}^{-1}$ ). In the left column we have represented the raw form factor $|\tilde{\rho}|^{2}$. In the right column, we have plotted $|\tilde{\rho}|^{2} / w_{0}^{4}$. With this representation, we can clearly notice the quadratic scaling with laser power [the yellow line of almost constant value in the left part of (g)], expected at low powers [more precisely when $w_{0} \ll 1 /\left(k_{m} r_{56}\right) \approx 1.26$ here]. Note that laser power is proportional to $w_{0}^{2}$. Parameters are identical to the ones used for Fig. 7 and the experimental results displayed in Fig. 6. For clarity, we have not represented the data in the extremely low wave number part [note that Eq. (7) implies that we must have $\tilde{\rho}^{2}(\bar{\nu})=1$ at $\bar{\nu}=0$ ]. More precisely, the sharp horizontal white regions near $\bar{\nu}=0$ correspond to $\tilde{\rho}^{2}>4 \times 10^{-3}$ in (d), and $\tilde{\rho}^{2} / w_{0}^{4}>1 \times 10^{-10}$ in (h).

This may be important in order to compare the maximum accessible output energy in the present case, and in the case of classical slicing. Besides, the theoretical study was performed assuming a plane wave for the laser. Further refined studies, taking into account the focusing of the laser, are also expected to add useful quantitative information, both on the actual form factor and also on possible localized transverse saturation effects.

\section{ACKNOWLEDGMENTS}

This work was supported by the Joint Studies Program of the Institute for Molecular Science, the JSPS fellowship program for research in Japan (Grant No. S-09171), and the Projet International de Coopération Scientifique (PICS) from CNRS. Part of this work was supported by Grant-inaid for scientific researches (B20360041) of JSPS, and the ANR (Blanc 2010-042301). The CERLA is supported by the French Ministère chargé de la Recherche, the Région Nord-Pas de Calais and the FEDER.

\section{APPENDIX: NUMERICAL CALCULATION OF HARMONIC CONTENT VERSUS LASER POWER}

At high power, the saturation effect is expected to be accompanied by an increase of harmonics in the emitted CSR. In the case of the experiment described in the previous sections, the computation shows that the level of harmonics is much lower than the fundamental (at the modulation wave number $k_{m}$ ). The data are summarized 
in Fig. 8, where we have displayed the form factor versus wave number and energy modulation. Two types of representation are used. In the left column, we have represented the raw data, and in the right column, we have represented the form factor divided by $w_{0}^{4}$ (which is proportional to the square of the laser power). This representation emphasizes the range of laser power (or equivalently energy modulation $w_{0}$ ) for which the $\mathrm{THz}$ power follows a quadratic scaling versus laser power.

[1] G. Carr, M. C. Martin, W. R. McKinney, K. Jordan, G. Neil, and G. Williams, Nature (London) 420, 153 (2002).

[2] T. Nakazato et al., Phys. Rev. Lett. 63, 1245 (1989).

[3] R. Schoenlein, S. Chattopadhyay, H. Chong, T. Glover, P. Heimann, W. Leemans, C. Shank, A. Zholents, and M. Zolotorev, Appl. Phys. B 71, 1 (2000).

[4] R.W. Schoenlein, S. Chattopadhyay, H. H. W. Chong, T. E. Glover, P. A. Heimann, C. V. Shank, A. A. Zholents, and M. S. Zolotorev, Science 287, 2237 (2000).

[5] S. Khan, K. Holldack, T. Kachel, R. Mitzner, and T. Quast, Phys. Rev. Lett. 97, 074801 (2006).

[6] J. M. Byrd, Z. Hao, M. C. Martin, D. S. Robin, F. Sannibale, R. W. Schoenlein, A. A. Zholents, and M. S. Zolotorev, Phys. Rev. Lett. 96, 164801 (2006).

[7] K. Holldack, T. Kachel, S. Khan, R. Mitzner, and T. Quast, Phys. Rev. ST Accel. Beams 8, 040704 (2005).

[8] M. Shimada, M. Katoh, S.-i. Kimura, A. Mochihashi, M. Hosaka, Y. Takashima, T. Hara, and T. Takahashi, Jpn. J. Appl. Phys. 46, 7939 (2007).

[9] S. Bielawski, C. Evain, T. Hara, M. Hosaka, M. Katoh, S. Kimura, A. Mochihashi, M. Shimada, C. Szwaj, T. Takahashi, and Y. Takashima, Nat. Phys. 4, 390 (2008).
[10] F. Sannibale, A. Marcelli, and P. Innocenzi, J. Synchrotron Radiat. 15, 655 (2008).

[11] C. Evain, C. Szwaj, S. Bielawski, M. Hosaka, Y. Takashima, M. Shimada, S. Kimura, M. Katoh, A. Mochihashi, T. Takahashi, and T. Hara, Phys. Rev. ST Accel. Beams 13, 090703 (2010).

[12] J. G. Neumann, R. B. Fiorito, P. G. O'Shea, H. Loos, B. Sheehy, Y. Shen, and Z. Wu, J. Appl. Phys. 105, 053304 (2009).

[13] D. Xiang and G. Stupakov, Phys. Rev. ST Accel. Beams 12, 080701 (2009).

[14] M. Dunning, C. Hast, E. Hemsing, K. Jobe, D. McCormick, J. Nelson, T. O. Raubenheimer, K. Soong, Z. Szalata, D. Walz, S. Weathersby, and D. Xiang, Phys. Rev. Lett. 109, 074801 (2012).

[15] M. Kumar and V. Tripathi, Phys. Plasmas 19, 073109 (2012).

[16] A. S. Weling and D. H. Auston, J. Opt. Soc. Am. B 13, 2783 (1996).

[17] C. J. Hirschmugl, M. Sagurton, and G. P. Williams, Phys. Rev. A 44, 1316 (1991).

[18] R. Piessens, E. De Doncker-Kapenga, and D. Kahaner, QUADPACK, A Subroutine Package for Automatic Integration (Springer, New York, 1983), p. 301; P Favati, G. Lotti, F. Romani, ACM Trans. Math. Softw. 17, 218 (1991) [http://www.netlib.org/quadpack/].

[19] A. Zholents and K. Holldack, in Proceedings of the FEL Conference, Berlin (2006), THPPH059, p. 725 [http://accelconf.web.cern.ch/AccelConf/f06/PAPERS/ THPPH059.PDF].

[20] K. Holldack, T. Kachel, S. Khan, R. Mitzner, and T. Quast, Phys. Rev. ST Accel. Beams 8, 040704 (2005).

[21] T. Tanikawa, M. Adachi, H. Zen, M. Hosaka, N. Yamamoto, Y. Taira, and M. Katoh, Appl. Phys. Express 3, 122702 (2010).

[22] M. Labat, N. Joly, S. Bielawski, C. Szwaj, C. Bruni, and M. E. Couprie, Phys. Rev. Lett. 103, 264801 (2009). 